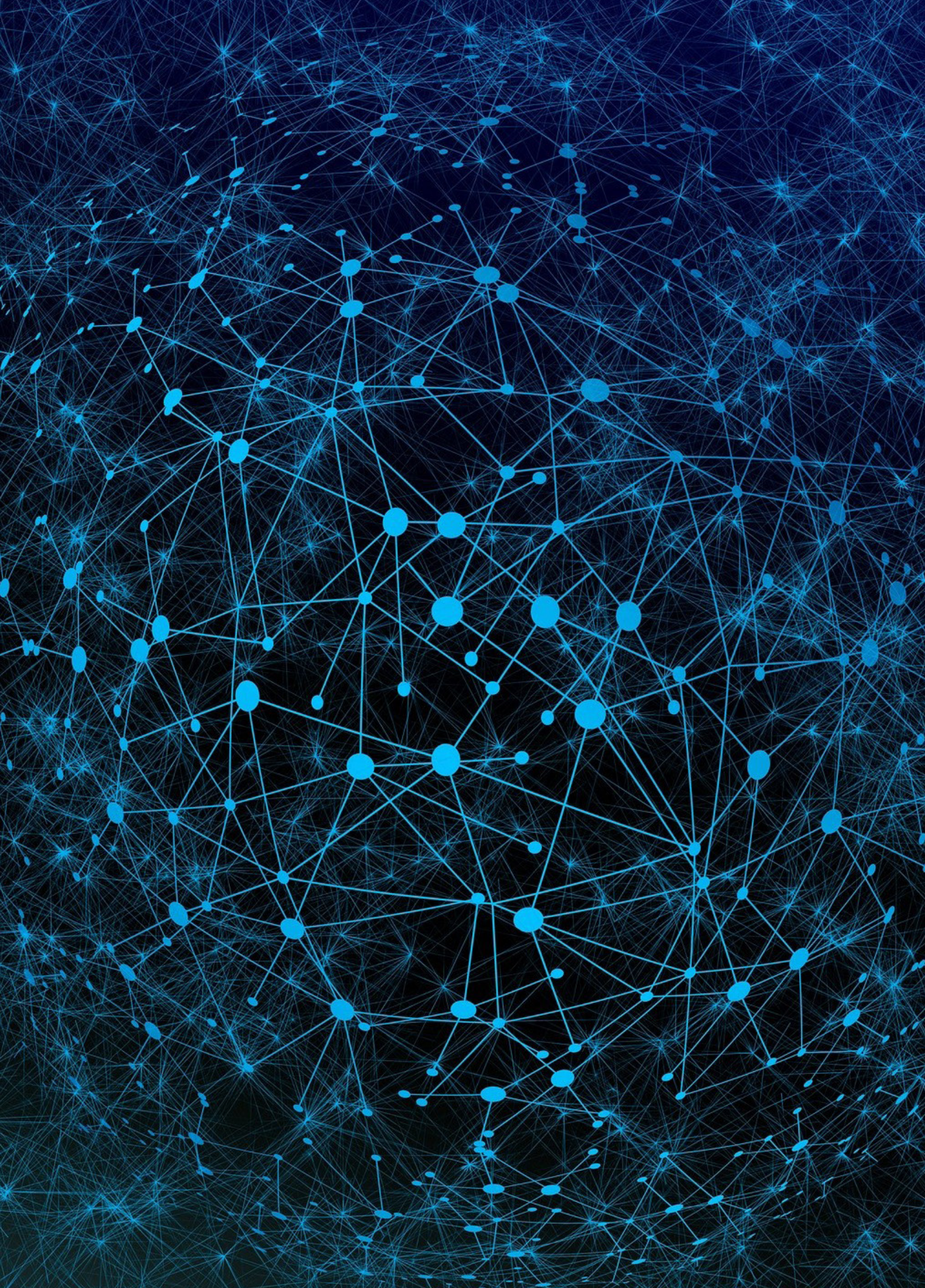




\title{
CONECTORES Y ANÁLISIS INFERENCIAL EN BÁSICA PRIMARIA
}

\section{Linking words and inferential analysis in primary school}

\author{
Lisbeth Nataly Pestana Velandia ${ }^{1}$, Hernán Martínez Ferro ${ }^{2}$ \\ ${ }^{1}$ Institución Educativa Técnica Industrial Antonio Ricaurte de Villa de Leyva, \\ 2Universidad Pedagógica y Tecnológica de Colombia \\ Email: ${ }^{1}$ lisbeth.pestana@uptc.edu.co, zhernan.martinez@uptc.edu.co
}

(Recibido el 28 de mayo de 2021 - Aceptado el 08 de junio de 2021)

\section{RESUMEN}

La inferencia es un proceso lógico-cognitivo que permite llegar a una proposición teniendo en cuenta como base inicial, otras proposiciones dadas. Por tanto, permite crear relaciones o conexiones entre los significados en un conjunto de proposiciones. La lectura inferencial es un momento imprescindible del proceso que conduce a la lectura crítica, al punto que se puede afirmar que sin lectura inferencial no se puede hacer lectura crítica. Teniendo en cuenta el anterior postulado, esta investigación se centró en fortalecer el análisis inferencial, a partir del uso de conectores discursivos en textos argumentativos, con niños de grado tercero. Para ello se escogieron tres conectores del discurso: "porque", "sin embargo" y "además". Con ellos se pretendió que el estudiante se familiarizara y entendiera el sentido de cada uno, y al usarlos adecuadamente, les permitiera entender la estructura de un argumento y fácilmente identificar las funciones que cumplen las premisas y las conclusiones dentro del mismo. Se implementó una propuesta bajo el paradigma interpretativo con un enfoque mixto de investigación, a través de la aplicación de unidades didácticas. Los resultados evidenciaron un mayor empleo de los conectores como guía para realizar inferencias, así mismo facilitaron la identificación de premisas y conclusiones, llevando a la creación de argumentos propios con relación lógica.

Palabras clave: argumentación, inferencia, lectura, conectores.

\section{ABSTRACT}

The inference is a logical-cognitive process that allows us to arrive at a proposition taking into account as an initial basis, other given propositions. Therefore, it allows creating relationships or connections between meanings in a set of propositions. Inferential reading is an essential moment in the process that leads critical reading, to the point that it can be said that without inferential reading, critical reading cannot be done. Based on the previous postulate, this research focused on strengthening inferential analysis, from 
the use of discursive connectors in argumentative texts, with third-grade children. For this reason, three connectors of the discourse were chosen: "because", "however" and "also". With them, it was intended that the student familiarize himself and understand the meaning of each one, and when using them appropriately, allow them to understand the structure of an argument and easily identify the functions that fulfill the premises and conclusions within it. A proposal was implemented under the interpretive paradigm with a mixed research approach, through the application of didactic units. The results showed a greater use of the connectors as a guide to make inferences, likewise, they facilitated the identification of premises and conclusions, leading to the creation of their own arguments with a logical relationship.

Keywords: argumentation, inference, reading, connectors.

\section{INTRODUCCIÓN}

Incentivar y fortalecer el ejercicio de la argumentación y la lectura crítica implica generar y promover espacios donde los estudiantes puedan expresar sus ideas y pensamientos libremente. Así mismo, integrar los saberes previos, involucrar a la familia y relacionar los temas trabajados con el entorno, posibilita que estos espacios promuevan el trabajo colaborativo y sean significativos en los procesos de enseñanza y aprendizaje, tanto del docente como de los estudiantes.

Como lo recomienda el Ministerio de Educación Nacional (MEN) de nuestro país, en los estándares básicos de competencias para el área de lengua castellana se pretende que:

El trabajo pedagógico que se adelante en el área debe incluir la generación de experiencias significativas para los estudiantes en las que se promueva la exploración y el uso de las diferentes manifestaciones del lenguaje verbales y no verbales, de tal forma que las asuman e incorporen, de manera consciente, intencional y creativa, en sus interacciones cotidianas y con diferentes fines: descriptivos, informativos, propositivos, expresivos, recreativos, argumentativos, entre otros. (2006, p. 28)

Así, el estudiante que posee un buen nivel de lectura crítica, desarrolla y practica habilidades mentales, competencias comunicativas como la discursiva y la textual, aplica el pensamiento crítico en diferentes ámbitos de su vida, lo que fortalece su rendimiento académico en las diferentes áreas del conocimiento. Según los lineamientos curriculares del Ministerio de Educación Nacional (1998): 


\section{(...) por lectura crítica ha de entenderse un saber proponer interpretaciones en profundidad} de los textos. La interpretación en profundidad implica un proceso de lectura que va desde el nivel primario, o lectura literal, pasa por un nivel secundario, o lectura inferencial y converge en un nivel crítico-intertextual. (p.53)

Por lo anterior, se hace necesario buscar estrategias metodológicas que fortalezcan la lectura inferencial y la lectura crítica, teniendo en cuenta que: "La lectura crítica no es la "libre opinión" del lector. La lectura crítica surge del ejercicio intelectual que presupone hacer inferencias, sean simples o complejas" (Jurado, 2014, p. 26). Pero, ¿Cómo fortalecer el análisis inferencial y la lectura crítica? Por esta razón, esta investigación apostó por promover la lectura inferencial, a partir del uso de conectores discursivos en textos argumentativos.

Para ello se propuso la implementación de unidades didácticas, en las que se les presentó a las participantes actividades en las cuales se pudiera identificar aspectos relacionados con el razonamiento, el uso de los conectores del discurso que fueron escogidos y que promovieran la realización de inferencias sencillas, identificando premisas y conclusiones. La implementación de las unidades didácticas se llevó a cabo con 10 niños y niñas de tercer año, de la Institución Educativa Técnica Industrial Antonio Ricaurte de Villa de Leyva - Boyacá. ${ }^{1}$

La investigación se enmarcó bajo el paradigma interpretativo y asumió el enfoque mixto de investigación. En la parte cualitativa, se buscó hacer la interpretación y descripción del análisis inferencial en textos argumentativos teniendo en cuenta el uso de los conectores discursivos en las cuatro unidades didácticas. En la parte cuantitativa se buscó observar con qué frecuencia los participantes eligieron cada uno de los tres conectores, tanto en la prueba diagnóstica inicial como final, con el fin de identificar cuál de ellos usaron con más frecuencia antes y después de la aplicación de las unidades didácticas.

En este artículo se presentarán tres partes de la propuesta de investigación: 1) el marco teórico conceptual; 2) las unidades didácticas; 3) análisis de resultados.

\section{MARCO TEÓRICO CONCEPTUAL}

En lo referente a la lectura inferencial, la investigación se fundamenta en la lógica proposicional de Irving Copi y Carl Cohen, en el desarrollo de las teorías de la argumentación que desarrollan Perelman y Toulmin a mediados del siglo XX y, en la propuesta de

\footnotetext{
${ }^{1}$ Se tuvieron en cuenta las consideraciones éticas pertinentes tales como el consentimiento informado y los permisos por parte de la Institución en la que se llevó a cabo la investigación.
}

Revista ingeniería, Investigación y Desarrollo (I2+D) Vol. 20 - No. 2, 2020 
filosofía para niños de Matthew Lipman. El reto principal con estos referentes teóricos consistió en derivar algunos elementos básicos de lógica y la argumentación que pudieran ser apropiados para introducir a los niños de tercer año en la lógica y la inferencia.

De la Introducción a la lógica de Irving Copi y Carl Cohen se retoman diferentes elementos: la definición de lógica como "el estudio de los métodos y principios utilizados para distinguir el razonamiento bueno (correcto) del malo (incorrecto)" (Copi y Cohen, 1995, p.17). La definición de argumento: “Desde el punto de vista lógico, un argumento es cualquier conjunto de proposiciones de las cuales se dice que una se sigue de las otras, que pretenden apoyar o fundamentar su verdad. Por supuesto, la palabra "argumento" se usa frecuentemente en otros sentidos, pero en lógica tiene el sentido que se ha explicado". (1995, p. 20)

Como la distinción entre premisas y conclusión es el nivel de análisis más básico del análisis inferencial, pero al mismo tiempo el nivel más importante para entender un argumento, la investigación optó por centrarse en esta distinción como la estrategia más apropiada para desarrollar la lectura inferencial en niños de tercer año. En este sentido, la idea básica que la investigación tomó de Copi y Cohen es que hay unos indicadores de premisas y unos indicadores de conclusión que se usan en el lenguaje común; si los niños son capaces de entender el sentido y uso de estos indicadores, entonces pueden diferenciar una premisa y una conclusión, lo que, a su vez, les permitiría ordenar un argumento.

Algunos indicadores de conclusión que señalan Copi y Cohen son: "por lo tanto", "de ahí que", "así", "correspondientemente", "en consecuencia", "consecuentemente", "lo cual prueba que", "como resultado", "por esta razón", "por estas razones", "se sigue que", "podemos inferir que", "concluyo que", "lo cual muestra que", "lo que nos permite inferir que" (1995, p. 24).

Algunos de los indicadores de premisa que señala Copi y Cohen son: "puesto que", "dado que", "a causa de", "porque", "pues", "se sigue de", "como muestra", "como es indicado por", "la razón es que", "se puede inferir de", "en vista de que" (1995, p. 25).

El uso de estos indicadores señala frecuentemente, pero no siempre, que lo que sigue es una conclusión o una premisa. Por este motivo, entender el uso de estos indicadores resulta valioso para comprender y organizar un argumento. Sin embargo, como la lista es bastante amplia y se está trabajando con niños de tercer año, la investigación tomó dos decisiones: primero, privilegiar el estudio de la lógica como un arte que se tiene que ejercitar y no como una ciencia teórica, en otras palabras, a los niños no se les hizo énfasis en que entendieran qué era una premisa, un argumento o una conclusión, se les ejercitó 
en el uso de ciertos conectores o indicadores; segundo, dado que la lista de indicadores de premisa y conclusión es bastante amplia, se eligió solo tres conectores "porque", "sin embargo" y "además", los dos últimos no son propiamente indicadores de premisa o conclusión, pero son conectores discursivos que expresan una conexión lógico-semántica entre dos enunciados, por lo que su manejo apropiado sirve para entender la estructura de un argumento o una inferencia. Como afirma Martínez (2014):

Los conectores son unidades léxicas, invariables y con un alto grado de lexicalización que expresan una relación conectiva lógico-semántica entre dos o más enunciados (o párrafos). Por tanto, su uso genera una serie de expectativas respecto a cómo orientar la información que introducen y por ello funcionan para el lector como pistas para la interpretación. (p. 23)

Dado que la investigación parte de la tesis de que el uso apropiado del lenguaje, oral y escrito, está directamente relacionado con la comprensión lógica de su construcción, la investigación revisó también los planteamientos de la teoría de la argumentación. En efecto, las teorías de la argumentación, que se desarrollaron en la segunda mitad del siglo pasado, parten del estudio de la misma, como una práctica comunicativa que utiliza el lenguaje natural con toda su riqueza y ambigüedad.

De la teoría de la argumentación desarrollada por Chaim Perelman, también llamada nueva retórica, se retoma la idea central, según la cual, la argumentación es una acción comunicativa en la que un orador pretende, mediante un discurso razonado, persuadir o convencer a un auditorio. Esto quiere decir que, la argumentación no es algo impersonal, sino que siempre está dirigida y, por lo mismo, adaptada al auditorio. Lo que implica la distinción entre dos usos de la racionalidad: un razonamiento formal, que es utilizado en las ciencias matemáticas en donde se puede encontrar una conclusión indiscutible, y una argumentación que pretende identificar buenos argumentos para ganar la adhesión de un auditorio. Por supuesto, el reto era hacer comprender a los niños, sin mencionarles ningún aspecto teórico; es decir, que hay campos como lo preferible, lo razonable y lo deseable en donde se debe argumentar y tratar de ganar la aceptación de los otros, aunque, hay otras argumentaciones que llegan a conclusiones diferentes. La solución que se encontró fue orientar a los niños en la discusión, en lo que con Lipman se denomina una comunidad de indagación, sobre asuntos en los que ellos encuentran tanto diferentes puntos de vista como respuestas a un mismo problema, con el objetivo de que tomaran partido por la solución que les pareciera más adecuada. De esta manera, sin entrar en la teoría, los niños entendían que hay asuntos para los cuales se encuentran diferentes soluciones, y de lo que se trata, es de seleccionar el que parezca más razonable y mejor argumentado. 
Como se menciona, la filosofía para niños de Matthew Lipman fue otro referente importante de la investigación. El programa de filosofía para niños y jóvenes intenta introducirlos en el mundo de las reglas básicas de la lógica formal, donde a través de la comunidad de indagación, ellos mismos, descubren, analizan, interpretan y aplican esas reglas para lograr dar respuesta a los diferentes problemas de su alrededor, de carácter, éticos, estéticos, críticos, etc. De Lipman, la propuesta trabajó la comunidad de indagación, ejercicio que permitiría a los niños centrarse en defender su tesis y buscar bases para sustentarla, por lo que está en consonancia con la pretensión de la teoría de la argumentación.

\section{UNIDADES DIDÁCTICAS}

En este apartado se presentan muestras de las actividades de la propuesta implementada en la investigación. Inicialmente, se encuentra el modelo estructural de las pruebas diagnóstica inicial y final; luego se presentan algunas actividades implementadas en cada una de las unidades didácticas.

Prueba diagnóstica inicial y prueba final

La Figura 1, muestra la estructura tanto de la prueba diagnóstica inicial como de la final, las cuales fueron diseñadas con el objetivo de observar la frecuencia del uso de cada uno de los conectores elegidos para la investigación. En concordancia, se plantearon 15 argumentos simples, es decir, una premisa y una conclusión que se debía articular a uno de los tres conectores.

\section{Figura 1.}

Prueba diagnóstica inicial

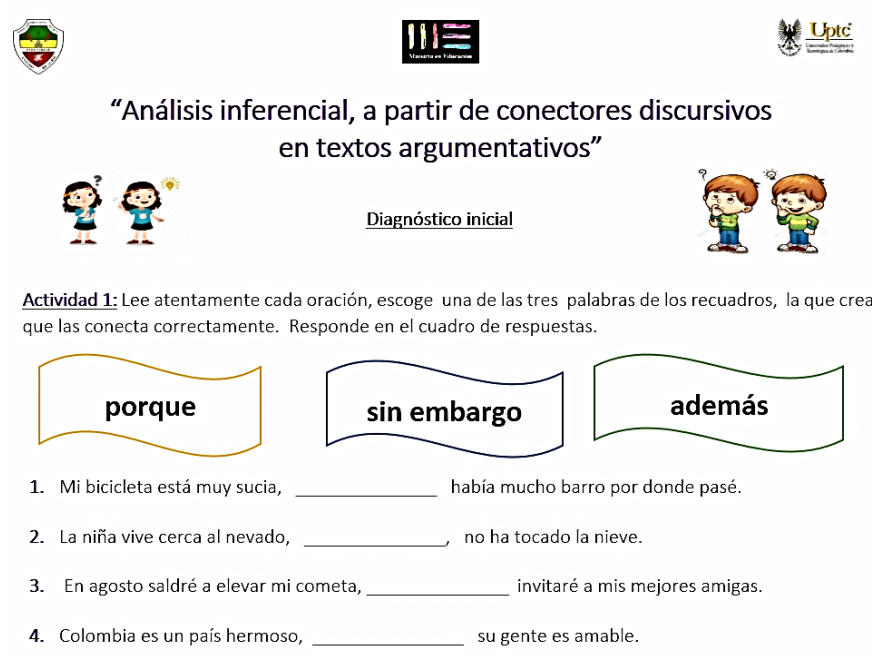

Fuente. Autores 


\section{Primera unidad didáctica: "Familiarización con los conectores"}

Esta primera unidad tuvo como fin acercar y familiarizar a los estudiantes con los conectores que se trabajarían en las siguientes tres unidades didácticas. En las Figuras 2 , 3 y 4 , se muestra parte de las actividades planteadas en las que se desarrollan ejercicios de creatividad, comparación y construcción.

\section{Figura 2.}

Ejemplo de actividad primera unidad didáctica

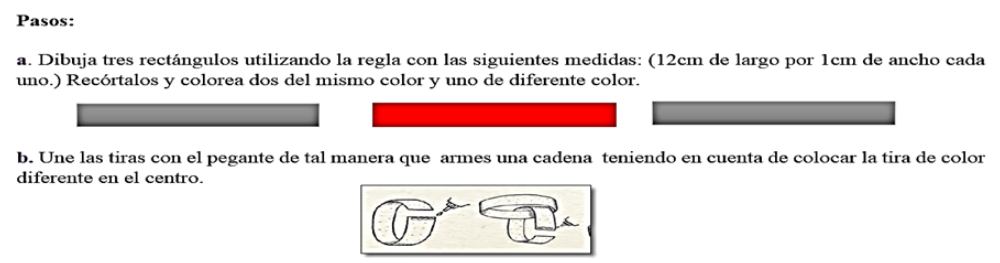

c. Cuando este armada tu cadena, imagina que la primera tira de la cadena, es una oración, la tira del medio que es de diferente color es una palabra que las une y se llama conector y la tercera tira es la otra oración con la que se relaciona la primera oración. Si es posible escribeles el nombre a cada tira. Así:

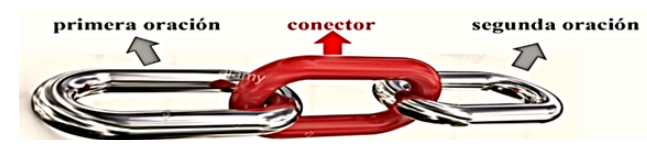

Fuente. Autores

\section{Figura 3.}

Ejemplo de actividad primera unidad didáctica

\section{"sin embargo"}

\section{Es un día caluroso, sin embargo, tengo frio}

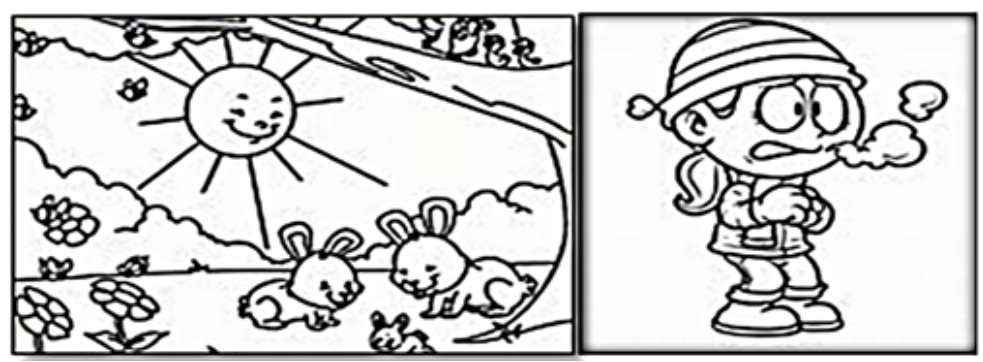

Fuente. Autores 


\section{Figura 4.}

Ejemplo de actividad primera unidad didáctica

3. Ejercicio de construcción:

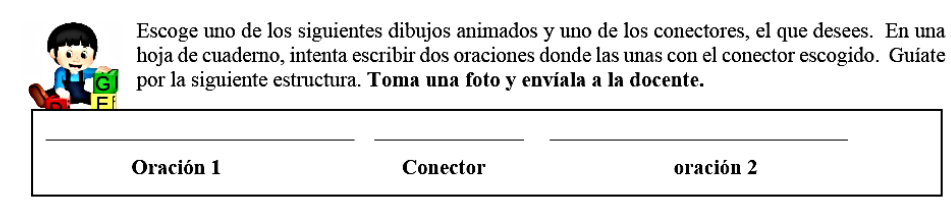

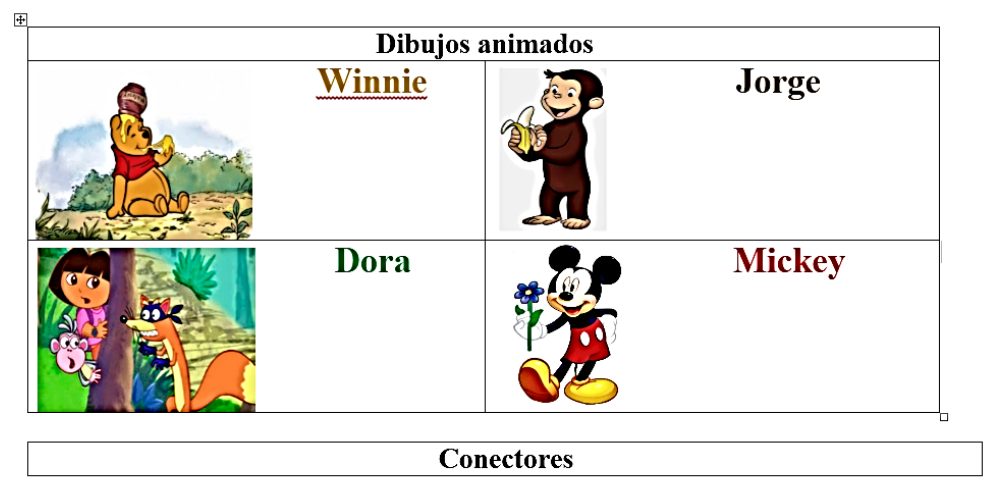

Fuente. Autores

Segunda unidad didáctica: "Saberes previos sobre los conectores"

Esta segunda unidad tuvo como fin identificar la concepción que los niños tenían de cada conector elegido. La Figura 5, muestra parte de las actividades desarrolladas para cumplir con el objetivo de la misma. De igual forma, se inició el ejercicio de preguntas para la comunidad gracias a la indagación con la familia, el cual relacionó temas transversales como normas, reglas, valores etc. Además, se incluyeron ejercicios para completar con los conectores.

\section{Figura 5.}

Ejemplo de actividades segunda unidad didáctica

\section{"El juego"}

$\underline{\text { Taller } 1}$

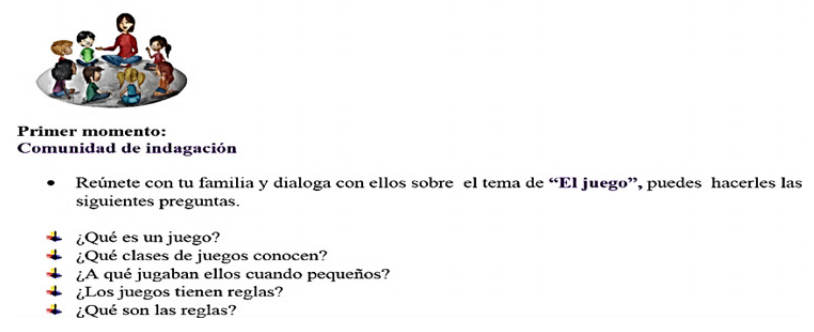




\section{Segundo momento: \\ Práctica con conectores}

- Lee las siguientes oraciones, escribe sobre la línea el conector que crees que las relaciona

correctamente y responde por qué escogiste ese conector. Ten en cuenta las imágenes encima de

cada conector, pues ellas te pueden recordar que significa o que te indican.
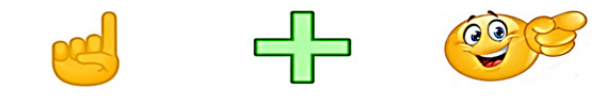

sin embargo

además

porque

1. A Maria le va muy bien en las carreras que participa, ¿Por qué escogiste este conector?

Fuente. Autores

Tercera unidad didáctica: "Ejercicios de ubicación y construcción"

Esta tercera unidad tuvo como fin describir la relación lógica que los estudiantes tenían al organizar palabras en desorden y armar argumentos con sentido y coherencia. Como consecuencia, La Figura 6, ilustra parte de las actividades desarrolladas para cumplir con el objetivo de la unidad.

\section{Figura 6.}

Ejemplo de actividades tercera unidad didáctica

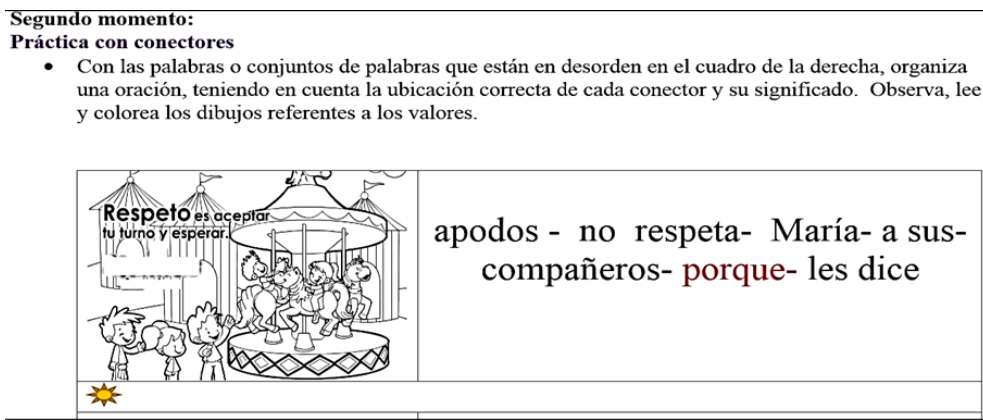

Ejercicio de construcción:

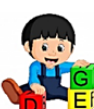

De acuerdo a lo que has aprendido respecto a los conectores, inventa tres oraciones, una para cada conector estudiado. Escríbelas en los siguientes cuadros siguiendo la estructura indicada.

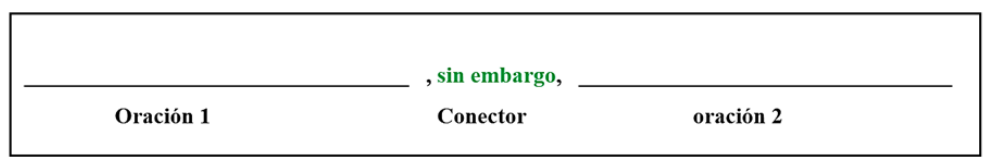

Fuente. Autores 
Cuarta unidad didáctica: "Ejercicios de comprensión y organización"

La cuarta unidad tuvo como fin evaluar la identificación de premisas y conclusiones en los diferentes ejercicios, teniendo en cuenta el sentido de cada conector.

La Figura 7, evidencia parte de las actividades desarrolladas para cumplir con el objetivo de la unidad, donde se presenta un ejercicio organizado para escoger la conclusión a la premisa dada en cada uno de ellos, todo relacionado con la lectura de un cuento.

\section{Figura 7.}

Ejemplo de actividad cuarta unidad didáctica

* sin embargo, no fue generosa con la cigarra.

* la hormiga trabajaba arduamente

* la cigarra pasó todo el verano cantando,

* además, burlándose de la hormiga porque trabajaba mucho.

* además, muchos animales no consiguen alimento.

* la hormiga tenía suficientes alimentos para compartir,

* porque necesitaba guardar alimentos para el invierno.

* en invierno hace mucho frio,

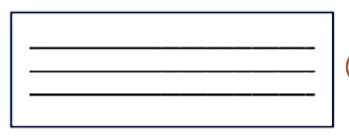

Oración 1

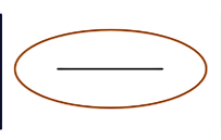

Conector

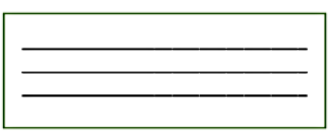

Oración 2

Fuente. Autores

\section{ANÁLISIS DE RESULTADOS Y CONCLUSIÓN}

Los resultados de la investigación se dividieron en dos partes. La primera, en la que se interpretaron los hallazgos de forma cuantitativa de la prueba diagnóstica, la final y la comparación de las dos pruebas; y una segunda parte, de forma cualitativa, que interpreta los resultados de los talleres de cada unidad didáctica frente al nivel inferencial que desarrollaron los estudiantes durante el proceso. Los siguientes resultados corresponden a la comparación que se realizó entre las pruebas (inicial y final) frente a la elección de cada uno de los conectores para dar respuesta a las 15 preguntas propuestas.

La Figura 8, muestra cómo la elección del conector "además" aumentó en un 12\% en comparación con la elección realizada de este mismo conector en la prueba diagnóstica inicial. 


\section{Figura 8.}

Elección del conector "además"

\begin{tabular}{|c|c|}
\hline \multicolumn{2}{|c|}{ Elección de conector "además" } \\
\hline $23 \%$ & $35 \%$ \\
\hline $\begin{array}{c}\text { PRUEBA } \\
\text { DIAGNÓSTICA } \\
\text { INICIAL }\end{array}$ & DIAGNÓSTICA \\
& FINAL = además \\
\hline
\end{tabular}

Fuente. Autores

La Figura 9, muestra cómo la elección del conector "sin embargo" aumentó en un 2\% en comparación con la elección realizada de este mismo conector en la prueba diagnóstica inicial.

\section{Figura 9.}

Elección del conector "sin embargo"

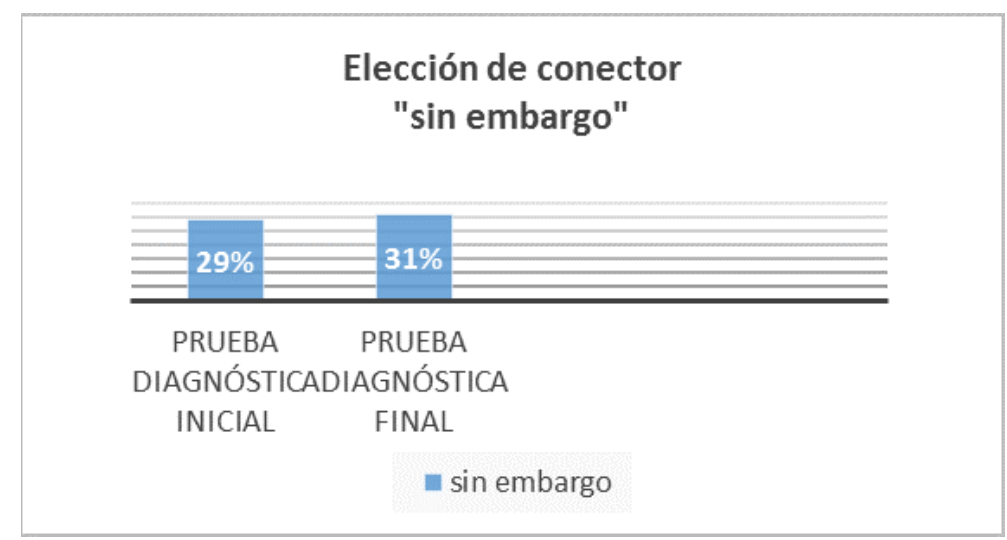

Fuente. Autores

La Figura 10, permite observar cómo el uso del conector "porque" disminuyó en un $14 \%$ en comparación con la prueba diagnóstica inicial, sin embargo, esto demuestra que los participantes sintieron más confianza y reconocieron el significado de los otros dos conectores y los eligieron con más frecuencia. 


\section{Figura 10.}

Elección del conector "porque"

\begin{tabular}{|c|c|c|}
\hline \multicolumn{3}{|c|}{ Elección de conector "porque" } \\
\hline $48 \%$ & $34 \%$ & \\
\hline $\begin{array}{l}\text { PRUEBA } \\
\text { DIAGNÓSTICA } \\
\text { INICIAL }\end{array}$ & $\begin{array}{c}\text { PRUEBA } \\
\text { DIAGNÓSTICA } \\
\text { FINAL }\end{array}$ & porque \\
\hline
\end{tabular}

Fuente. Autores

La Figura 11, da cuenta de las respuestas correctas totales de cada una de las pruebas presentadas por los participantes. En la misma, se evidencia que la cantidad de respuestas correctas en la prueba diagnóstica inicial fue mayor que en la prueba final, donde el porcentaje en el cual disminuyó la cantidad de respuestas acertadas correspondió a un $4 \%$. Sin embargo, este resultado da pie para preguntarnos ¿qué sucedió?, ¿por qué el porcentaje de respuestas acertadas no aumentó en la última prueba después de aplicadas las unidades didácticas?, ¿este tipo de test sí responden verdaderamente al aprendizaje o conocimientos adquiridos por parte de los participantes? Estos resultados contradicen a los observados directamente en la implementación de cada una de las unidades didácticas, donde se evidenció la evolución en el proceso.

\section{Figura 11.}

Respuestas acertadas de cada test

\section{Respuestas acertadas en cada test}

Fuente. Autores

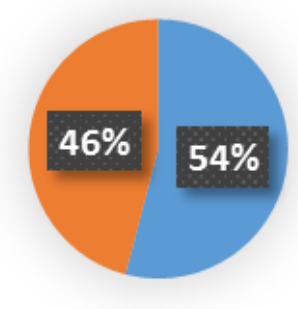

- Test inicial

- Test final 


\section{PRINCIPALES HALLAZGOS EN LOS RESULTADOS DE LAS UNIDADES DIDÁCTICAS}

Las cuatro unidades didácticas se organizaron de tal manera que los resultados de cada una, fueran consecuentes y dieran pie para la realización de las demás. En este proceso, se tuvo en cuenta lo más relevante y, por ende, las dificultades que se iban presentando a lo largo de la aplicación. Los principales hallazgos encontrados en la implementación de las unidades didácticas fueron:

\section{Primera unidad didáctica: Familiarización con los conectores}

En las actividades planteadas en esta primera unidad, se encontró que así los participantes no tengan claro una respuesta, siempre tienen en cuenta sus saberes previos como: "el factor más importante que influye en el aprendizaje es lo que el alumno ya sabe. Averígüese esto y enséñesele en consecuencia". (Ausubel, Novak y Hanesian, 1983, como se citó en Rivera, D., 2000), así también, su contexto para relacionarlos, tomándolos como base para responder.

En las actividades donde se transversalizaron las temáticas, los participantes respondieron de manera acertada a la mayoría de las preguntas. En este ejercicio, se logró identificar la facilidad para reconocer el significado de cada conector de acuerdo con la temática con la que fue comparado. Por otro lado, cuando se les presentaron argumentos solo con premisa y el conector "porque," los participantes mantuvieron la misma línea temática y lograron dar conclusión a los ejercicios dados en un 100\%. Sin embargo, en muchas de las respuestas se evidenciaron dificultades en la escritura como palabras unidas y faltas ortográficas.

Las actividades con imágenes fueron las que mejor se respondieron y en las que menos se presentó dificultad. El conector "porque" fue el más utilizado por los participantes después de la prueba diagnóstica inicial y el "además" el menos utilizado. Por otro lado, el conector "sin embargo" fue utilizado muy poco por los participantes y en las oraciones que lo emplearon no se evidenció una clara coherencia.

Segunda unidad didáctica: Saberes previos sobre los conectores

Esta segunda unidad se planteó con el fin de Identificar la concepción que los niños tenían de cada conector escogido para trabajar. En ella se encontró que al proponer argumentos incompletos sin conector que relacionara la premisa y la conclusión, los participantes mostraron mínima de dificultad para elegir el más adecuado. En el ejercicio donde se les planteó seis argumentos incompletos a los diez participantes, 49 de las 60 respuestas fueron acertadas correspondiendo al 81,6\% y, 11 respuestas erradas correspondientes al 18.33\%. Respecto a la elección de cada conector, se observó que el conector "porque" 
continuó siendo el predilecto por los participantes. En el caso de los otros dos conectores, el "sin embargo" disminuyó su nivel de elección, pero dio paso al conector "además", el cual poco se había elegido en la primera unidad didáctica. En lo que se refiere a las concepciones sobre el significado de cada conector, se observó que se les dificulta emitir una concepción clara, sin embargo, presentan baja dificultad para emplearlo de acuerdo con el contexto expuesto en el argumento.

\section{Tercera unidad didáctica: Ejercicios de ubicación y construcción}

Para esta tercera unidad didáctica se describió la relación lógica que los estudiantes tuvieron al organizar palabras en desorden y armar argumentos con sentido, teniendo en cuenta la ubicación de la premisa, el conector y la conclusión; además, se quiso comprobar el sentido que los participantes le daban a cada conector para escribir oraciones propias. En los resultados analizados en la primera actividad, se encontró que la cantidad de argumentos organizados igual al modelo o respuesta correcta fueron 23 de los 50 en total, y que en las otras 27 respuestas, se identificaron aspectos como organización de argumentos diferentes al modelo pero con una relación lógica y con sentido; así también, se encontró omisión de los conectores y presentación de oraciones simples sin relación entre ellas; organización de la premisa y conector pero con una conclusión que quitaba el sentido a todo el argumento; respuestas similares al argumento presentado en desorden y, organización de respuestas sin conector intentado dar respuesta a una pregunta inexistente.

En lo que se refiere a la segunda parte de esta unidad, donde se dio vía libre a la creación de argumentos teniendo en cuenta cada uno de los tres conectores, se encontró que de los 30 argumentos creados por los participantes (10 por cada conector). Fue posible identificar que 7 argumentos en los que se utilizó el conector "sin embargo" evidenciaron una relación lógica, coherente que mantuvo la misma línea temática; en los otros 3, se intentó crear un argumento coherente, sin embargo, no se estructuraron muy bien o faltaron palabras para lograrlo. Por otro lado, en los argumentos creados con el conector "porque", 10 de los 10 presentaron relación lógica entre las partes del argumento. En lo que se refiere a los argumentos creados con el conector "además", 8 de los 10 presentaron relación lógica y coherente; y en los otros dos, la conclusión no mantuvo la misma línea temática.

Al realizar un balance general se encontró que 25 de los argumentos creados por los participantes evidenciaron relación lógica y coherente, donde el conocimiento del significado o sentido brindado por el conector, llevó a realizar un análisis inferencial para poder organizar una conclusión a la premisa propuesta que mantuviera la misma línea temática. 


\section{Cuarta unidad didáctica: Ejercicios de comprensión y organización}

En esta unidad se buscó evaluar la capacidad de los participantes para identificar premisas de conclusiones a través de temáticas relacionadas con lecturas. En la primera actividad se encontró que, de las 30 respuestas para completar los argumentos, en los cuales debían identificar la conclusión teniendo en cuenta el conector, 20 de las 30 fueron acertadas. En la segunda actividad, los participantes debían escoger premisas y conclusiones mezcladas entre sí, y con ellas estructurar argumentos con una relación lógica. El 67\% de los argumentos fueron estructurados correctamente, identificando y ubicando la premisa, el conector, y la conclusión. Un $28 \%$ de los argumentos presentaron intercambio de la premisa y la conclusión; y el otro 5\% no fueron organizados.

\section{CONCLUSIONES}

La inferencia es un ejercicio que se puede trabajar en cualquier área académica, además, se presta para que a través de diversas actividades se pueda promover su práctica. Cabe resaltar que el análisis inferencial es un proceso altamente significativo, y que los conectores discursivos facilitaron y promovieron este proceso de forma más práctica y didáctica. Así, el "pensamiento lógico de los niños se desarrolla más cuando en el proceso se tiene en cuenta la actividad creativa". (Lipman. 1992, p. 143)

A pesar de las particularidades y dificultades en tiempo de pandemia por el CO-VID-19, los participantes demostraron su capacidad de trabajo autónomo, pues, a pesar de las circunstancias y poca comunicación, lograron responder lo mejor posible a las diferentes actividades propuestas. Las unidades didácticas posibilitaron implementar las actividades de forma progresiva y ordenada, lo cual permitió poder hacer un análisis de la misma manera, ya que "es una forma de planificar el proceso de enseñanza-aprendizaje alrededor de un elemento de contenido que se convierte en eje integrador del proceso, aportándole consistencia y significatividad (Federación de enseñanza de CC.OO, 2010, p.1).

Durante el trabajo con los conectores elegidos para la investigación, se observó que el más reconocido e implementado por los participantes fue el "porque", ya que presenta una alta preponderancia y manejo al hablar y escribir en sus actividades diarias como estudiantes, además, se les facilita describir porque sucede algo, es decir pueden crear conclusiones como consecuencias de una acción. No obstante, en el caso del conector "además" y el "sin embargo" se necesita seguir trabajando con el correcto empleo de estos, pues, aunque su uso no fue del todo bajo, sí existen vacíos y confusiones todavía. 
En efecto, se evidenció que el empleo correcto y el reconocimiento del significado de los conectores, posibilita eficazmente las inferencias que pueden hacer los participantes, y así mismo, estos permiten diferenciar la premisa de la conclusión en un argumento porque "poseen un cometido coincidente en el discurso: el de guiar, de acuerdo con sus distintas propiedades morfosintácticas, semánticas y pragmáticas, las inferencias que se realizan en la comunicación" (Portolés, 1998, pp. 25-26)

El uso de imágenes y textos llamativos posibilitó una mayor comprensión de las actividades planteadas pues "la imagen leída es portadora de sentido pues ofrece resonancias con la propia narración interna, para conectar con todas las posibilidades de interpretación: personal, contextual, social, cultural y simbólica" (Lenis, B. 2019, p. 36).

Durante el proceso de aplicación de los diferentes talleres de las unidades didácticas, se enseñó implícitamente ortografía y signos de puntuación, elementos valiosos para la comprensión textual. Así también, cuando se da ejemplo de la estructura de un argumento, es importante reconocer en dónde va incluido un conector.

Por otro lado, se considera que el tipo de prueba con respuestas cerradas, no siempre permite evidenciar el aprendizaje de un participante, como sí se puede evidenciar en el análisis inferencial elaborado durante todas las actividades de las unidades didácticas, en la invención de argumentos propios de manera coherente y con sentido, en los procesos cognitivos puestos en marcha para identificar información explícita, así mismo, en las relaciones lógicas dadas entre la premisa y la conclusión, donde los conectores jugaron un papel importante.

Se pudo demostrar que a través de la implementación de los conectores se puede fortalecer el análisis inferencial, es decir la "relación entre las premisas presentadas, afirmando una proposición con base en la otra como lo plantea (Copi. 2013, p, 7) y la expresión escrita con sentido coherente en participantes de básica primaria, ya que 'la inferencia hace parte de las operaciones mentales que se involucran con la comprensión lectora, y se basa principalmente en la articulación de información que debe ser reconstruida a partir de la relación de conocimientos y saberes internos y externos' (Navarro, 2009). Así mismo, la identificación y creación de argumentos propios, teniendo en cuenta que 'Un argumento es un conjunto de proposiciones del cual se dice que una de ellas se sigue de las otras, consideradas como base o fundamento para la verdad de este'," (Copi. 2013, p. 7).

Finalmente, aunque la aplicación de la propuesta de manera remota, presentó muchas dificultades de comunicación con los participantes, sí permitió ver en estos inconvenientes, puntos de partida creativos, logísticos, pensados especialmente en las condiciones del 
contexto, necesidades y particularidades de los participantes, pensando siempre en buscar la forma más fácil para poder llegar a ellos con la propuesta.

\section{REFERENCIAS}

Álvarez, A. y Álvarez, V. (2014). Métodos en la investigación educativa. México: Horizontes educativos.

Campos, G. y Lule, N. (2011, 09, 05). La observación, un método para el estudio de la realidad. Xihmai VII (13), 45-60, Enero-junio de 2012, fi-le:///C:/Users/NATALY\%20P/ Downloads/Dialnet-LaObservacionUnMetodoParaEIEstudioDeLaRealidad-3979972.pdf

Copi, I., y Cohen, C. (2013). Introducción a la lógica. Limusa

Federación de enseñanza de CC.OO. de Andalucía. (2010). Temas para la educación. № 7, https://www.feandalucia.ccoo.es/docu/p5sd6953.pdf

Jurado, $(4,05,2018)$. La lectura crítica: el diálogo entre los textos. Ruta maestra. Número 8, https://rutamaestra.santillana.com.co/wp-content/uploads/2018/05/La-lecturacr\%C3\%ADtica-el-di\%C3\%A1logo-entre-los-textos.pdf

Lenis, B.(2019). Inferencias en el proceso de lectura: imagen y palabras. (Tesis doctoral). Universidad ICESI. Santiago de Cali Colombia.

Lipman, M.; Sharp, A. \& Oscanyan F. (1992). la filosofía en el aula. Madrid. La torre.

Martínez, H. (2013). Legitimidad, razón y derecho: dos modelos de justificación política. Universidad Externado de Colombia.

Martínez, H. (2013). Legitimidad, razón y derecho: dos modelos de justificación política. Bogotá: Universidad Externado de Colombia.

Ministerio de Educación Nacional. (1998). Serie de lineamientos curriculares. Primera edición. http://www.socialhizo.com/.../legislacion/lineamientos-curriculares-de-lenguacastellana.

Ministerio de Educación Nacional. (2006). Estándares básicos de competencias en lenguaje, matemáticas, ciencias y ciudadanas. Primera edición de: https://www. mineducacion.gov.co/1621/articles-340021_recurso_1.pdf 
Navarro, M. (2009). Interpretar y argumentar la hermenéutica gadameriana a la luz de las teorías de la argumentación. Plaza y Valdez Editores.

Portolés, J., (1998). Marcadores del discurso. Barcelona: Ariel.

Algunos comentarios sobre la enseñanza de los marcadores del discurso escrito a estudiantes de ELE, en Carabela, Madrid, SGEL.

Quevedo, R. (2002). Introducción a la metodología de investigación cualitativa. Revista de psicodidáctica, volumen 14, https://www.researchgate.net/publication/39219263_ Introduccion_a_la_metodologia_de_investigacion_cualitativa

Real Academia Española. (2020). Test. En Diccionario de la lengua española (edición del tricentenario). Consultado el 26 de septiembre del 2020. https://dle.rae.es/test

Rivera, D. (2000). ¿Cuán importantes son los conocimientos previos, como punto de partida para el aprendizaje de habilidades psicomotrices en el subdirector de educación tecnológica? Horizontes Educacionales, núm. 5, 2000, pp. 75-80. https://www.redalyc. org/pdf/979/97917880010.pdf

Sandoval, C. (2002). Investigación Cualitativa. Bogotá: ARFO Editores e Impresores Ltda.

Toulmin, S.; Rieke, R. y Janik, A. (2018). Una introducción al razonamiento. (trad. José Gascón). Palestra Editores. (Original publicado en 1984) 